\title{
Variational Mode Decomposition Features for Heartbeat Classification
}

\author{
Amalia Villa ${ }^{1}$, Sibasankar Padhy ${ }^{1}$, Rik Willems ${ }^{3}$, Sabine Van Huffel ${ }^{12}$, Carolina Varon ${ }^{1}$ \\ ${ }^{1}$ KU Leuven, Department of Electrical Engineering-ESAT, STADIUS Centre for Dynamical \\ Systems, Signal Processing and Data Analytics, Leuven, Belgium \\ ${ }^{2}$ IMEC, Leuven, Belgium \\ ${ }^{3}$ Division of Experimental Cardiology, Department of Cardiovascular Diseases, KU Leuven, Belgium
}

\begin{abstract}
In software applications to analyse Heart Rate Variability $(H R V)$ or to detect heart rhythm disorders, automatic heartbeat classification is a first step to expose abnormalities in the electrical activity of the heart.

We propose a new morphological description of heartbeats based on Variational Mode Decomposition (VMD) to classify them as normal, supraventricular or ventricular. The proposed approach combines the features extracted from the different modes with time features, and it is designed for single-lead applications. The features are fed to an LS-SVM classifier, using an RBF kernel, 10-fold cross-validation and 50\% of balanced data as training. In this study, two different approaches were tested: one considering an intra-patient approach inspired by a semisupervised application, in which the same patients form the training and the test set; and a second inter-patient approach, in which the training and the testing signals belong to different patients.

The method reports an average accuracy of $92.17 \%$ and sensitivities of $92.84 \%, 72.56 \%$ and $91.25 \%$ for normal, supraventricular and ventricular beats respectively, which is in line with the state of the art.
\end{abstract}

\section{Introduction}

Heartbeat classification is a required step for several applications used for long-term electrocardiogram (ECG) analysis. For instance, the analysis of heart rate variability (HRV) should in principle use only the time intervals between normal beats. Another example is the automatic detection of arrhythmia, which can assist the diagnosis of heart diseases.

The classification of heartbeats is highly time consuming and tedious if done manually, and therefore, there is a big interest in automatic algorithms. The features used for this automatisation are very relevant, since they directly affect the results of the classifier. Previously de- scribed features are based on timing [1,2]; ECG morphology [3], wavelets [4], Principal Component Analysis (PCA) [5] or Independent Component Analysis (ICA) [6]. These features can be extracted either from single or multilead ECG.

This study focuses on feature extraction, aiming to find characteristics applicable to wearable devices, where a single-lead approach is used and the signals can generally be affected by noise. Here, the use of Variational Mode Decomposition (VMD) is proposed as a way to improve the information contained in single-lead ECG, while being a robust solution against noise.

\section{Methodology}

\subsection{Data}

In this study, the first lead of the MIT-BIH Arrhythmia dataset recordings was used for analysis [7]. The dataset consists of 48 recordings of approximately 30 minutes, sampled at $360 \mathrm{~Hz}$ and from which the R peak locations and the labels of the heartbeats are provided. AAMI recommendations suggest classifying heartbeats in 5 groups: Normal, Supraventricular ectopic beat, Ventricular ectopic beat, Fusion beat and Unknown beat. We considered only the first three classes. Table 1 lists the types of annotations from the MIT-BIH Arrhythmia dataset that are included in each of the three classes.

Following the recommendations in [7], four pacemaker patients were removed from the experiment. The 44 remaining registrations were divided in two sets of signals, DS1 and DS2, as specified in [1], to be able to compare the present results with the literature. Two approaches were considered regarding the data used for training and testing in the experiments performed, as suggested in [8]. On one hand, an intra-patient approach, as an indicator for a semisupervised version of the algorithm in which the same patients are used for training and testing. On the other hand, an inter-patient test, in which a set of patients is used for training and another set is used for testing. 
Given the fact that the three classes are very different in size in the present database, the training set needs to be balanced for the experiments performed. For the intrapatient approach, 472 heartbeats of each class are used for training, corresponding to half the size of the supraventricular class, which is the smallest one. The remaining heartbeats of the DS1 set are used for evaluating the algorithm. For the inter-patient approach, the training set consists of 944 heartbeats of each class from DS1, and the method is evaluated on the DS2 set. K-medoids is used in both approaches to select the training heartbeats of the ventricular and normal classes, in order to guarantee a good representation of the dataset.

\subsection{Preprocessing and Segmentation}

ECG signals are affected by several kinds of artefacts and noise, such as power-line interference, muscle artefacts, electrode movement or baseline wander. The method proposed in [1] for preprocessing is used in this approach, in which the baseline wander is removed with two consecutive median filters of $200 \mathrm{~ms}$ to extract the QRS complex and the $\mathrm{P}$ wave, and $600 \mathrm{~ms}$ for the $\mathrm{T}$ waves. The resulting signal is removed from the original one, and the outcome is fed into a 8 -order FIR low-pass filter with a $40 \mathrm{~Hz}$ cut-off frequency.

Regarding segmentation, this method requires a previous stage of $\mathrm{R}$ peak detection, since for the given experiments the annotated $\mathrm{R}$ peaks were used. Once the $\mathrm{R}$ peaks are located, an asymmetric window of $650 \mathrm{~ms}$ is applied to segment the heartbeats, as used in [9].

\subsection{Feature Extraction}

\subsubsection{VMD features}

Variational Mode Decomposition (VMD) is a method to adaptively decompose a signal into its principal modes. Those modes are selected considering that (1) they must be able to reconstruct the signal in an optimal way and (2) they are band-limited around an automatically estimated center frequency [10].

These characteristics make the method very appropriate for the present application, due to the quasi-stationary properties of the ECG, the robustness of the method against noise and the decomposition in a number of $k$ modes that improves the information of single-lead ECG. Furthermore, the center frequencies associated to each of the $k$ modes can be related to the different characteristic waves of the ECG: the P, QRS and T wave.

Being a parameterized method, VMD requires two parameters to be fixed in advance: the number of modes $k$ and the bandwidth control parameter $\alpha$. For ECG, the optimization technique developed by Guo et al. [11] leads to a
Table 1. Types of beats from the MIT-BIH Arrhythmia dataset included in classes Normal, SVEB and VEB.

\begin{tabular}{ccc} 
& Type of heartbeat & Symbol \\
\hline \hline \multirow{3}{*}{ Normal } & Normal Beat & $\mathrm{N}$ \\
& Left Bundle Branch Block (LBBB) & $\mathrm{L}$ \\
& Right Bundle Branch Block (RBBB) & $\mathrm{R}$ \\
\hline \multirow{4}{*}{ SVEB } & Atrial Premature Beat (APB) & $\mathrm{A}$ \\
& Aberrated APB & $\mathrm{a}$ \\
& Nodal/Junctional Prem. Beat & $\mathrm{J}$ \\
& Prem./Ectopic Supraventr. Beat & $\mathrm{S}$ \\
& Atrial Escape Beat & $\mathrm{e}$ \\
VEB & Junctional Escape Beat & $\mathrm{j}$ \\
\hline \hline & Ventricular Premature Beat & $\mathrm{V}$ \\
& Ventricular Escape Beat & $\mathrm{E}$ \\
\hline
\end{tabular}

number of modes $k$ of 5 and $\alpha$ of 30 .

Once the five modes are extracted for each of the heartbeats, they are sorted according to their central frequency. The five frequency bands selected are (1) from 0.5 to $3 \mathrm{~Hz}$, (2) from 3 to $6 \mathrm{~Hz}$, (3) from 6 to $9 \mathrm{~Hz}$, (4) from 9 to $13 \mathrm{~Hz}$ and (5) from 13 to $25 \mathrm{~Hz}$. The first four bands are based on the literature, since they correspond approximately to the heart rate $(1 \mathrm{~Hz}), \mathrm{T}$ wave $(4 \mathrm{~Hz}), \mathrm{P}$ wave $(7 \mathrm{~Hz})$ and $\mathrm{QRS}$ complex $(10 \mathrm{~Hz})$ [12]. Moreover, the last frequency band also presents information in the frequency spectrum of the ECG which can be used for classification.

The extraction and sorting of the modes result in a set of five modes per heartbeat as shown in Figure 1. As it can be observed, the three beat classes show differences in the morphology of the modes. Based on these differences, five features are extracted from each of the modes, and they are listed in Table 2.

As shown in the figure, the number of zero crossings and the amplitude difference between the deepest minima seem to differ among the three classes, while the symmetry around the $\mathrm{R}$ peak is specially considered to differ between Supraventricular and Ventricular heartbeats. In addition, the bandwidth at $3 \mathrm{~dB}$ and the total power in each band are extracted, leading to 25 morphological features extracted from all the modes.

\subsubsection{Temporal Features}

Since one of the most relevant aspects of an irregular beat is its timing, the four standard temporal features listed in Table 2 are also considered. These temporal features are normalized per patient, as recommended in [2].

\subsection{Classifier}

The 29 features, consisting of 25 VMD related features and 4 related to timing, are fed to an LS-SVM classifier using an RBF kernel[13]. Additionally, 10-fold crossvalidation is used to tune the parameters of the kernel. 


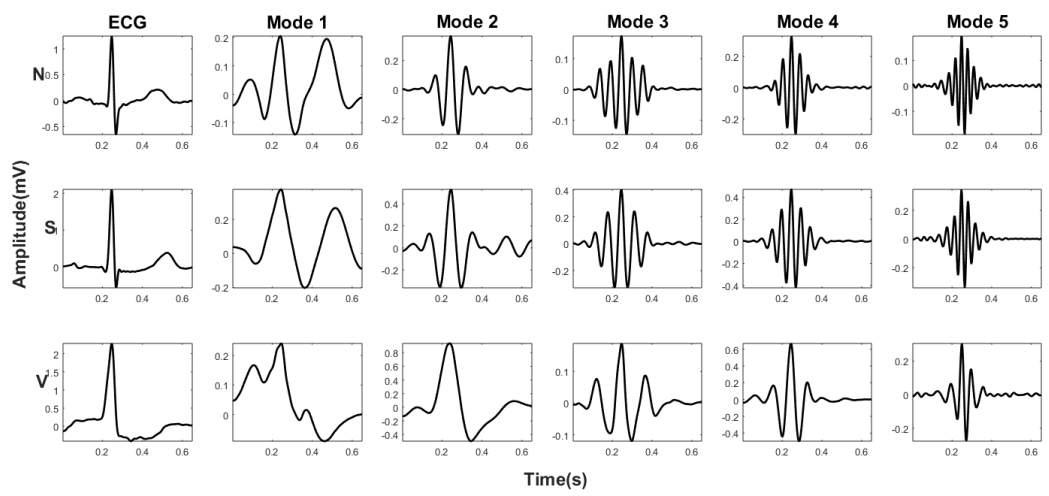

Figure 1. Example of Normal, Supraventricular and Ventricular beat: ECG and the 5 modes obtained.

Table 2. VMD and time features considered in the study. VMD Features

\begin{tabular}{c} 
Number of zero crossings \\
Symmetry around the R-peak \\
Amplitude difference between deepest minima \\
Bandwidth at $3 \mathrm{~dB}$ \\
Total Power in the bandwidth \\
\hline
\end{tabular}

Time Features

\section{Distance to previous $\mathrm{R}$ peak \\ Distance to next $\mathrm{R}$ peak}

Absolute difference between previous and post RR interval

Deviation of the RR distance to the last 10 intervals

\section{Results and discussion}

\subsection{Intra-patient results}

In this experiment, representative of a semi-supervised approach, the group of 22 records DS1 was used both for training and testing. Results are shown in Table 3, for the case of using time features, VMD or both. The best overall accuracy is $95.74 \%$, which was obtained with the combination of both types of features. Moreover, the use of time features allows to distinguish between normal and irregular heartbeats, while VMD features, which are more related to morphology, but ignore the presence of arrhythmia, assist in the distinction between supraventricular and ventricular heartbeats. The use of both kinds of features resulted in sensitivities above $90 \%$ for each of the classes. However, the specificity of supraventricular heartbeats still needs to be improved.

\subsection{Inter-patient results}

This experiment relates to the general problem in which the algorithm is trained with a set of patients and tested on different ones. Table 4 shows the results obtained for this approach. When using only VMD features, the sensitivity of ventricular heartbeats increased to $81.11 \%$, while when using temporal features, only $76.35 \%$ was obtained. In addition, the combination of both types of features led to an improvement in the results of every class. For normal heartbeats, the False Alarm Ratio was 3.75\%, with a speci- ficity of $99.59 \%$, and a sensitivity of $92.84 \%$. Ventricular heartbeats obtained a sensitivity of $91.25 \%$ and specificity of $80.84 \%$. For the supraventricular beats, the most challenging class, a sensitivity of $72.56 \%$ was obtained with the combination of VMD and temporal features, which was much higher than that obtained exclusively with one type of feature. In spite of this, the specificity for this class is still poor and requires more research to be improved.

\section{Conclusion}

This study proposed the use of Variational Mode Decomposition (VMD) as a new source of features for heartbeat classification. Three of the classes suggested by AAMI were considered: normal, ventricular and supraventricular. The new features, combined with traditional temporal features, were shown to obtain good results for the distinction of these three classes using single-lead ECG. While temporal features assist IN the detection of irregular heartbeats, VMD allows the differentiation of the type of irregularity. Despite the promising results, the method needs to be optimized to properly exploit the properties of the different modes.

\section{Acknowledgements}

Agentschap Innoveren \& Ondernemen (VLAIO): STW 150466 OSA+. European Research Council. The research leading to these results has received funding from the European Research Council under the European Unions Sev- 
Table 3. Results for the intra-patient approach for time features, VMD features and both.

\begin{tabular}{|c|c|c|c|c|c|c|c|c|c|c|}
\hline & \multirow{2}{*}{$\begin{array}{c}\text { Overall } \\
\text { Accuracy }\end{array}$} & \multicolumn{3}{|c|}{$\mathrm{N}$} & \multicolumn{3}{|c|}{$\mathrm{Sv}$} & \multicolumn{3}{|c|}{ V } \\
\hline & & $\mathrm{Se}$ & $\mathrm{P}+$ & FPR & $\mathrm{Se}$ & $\mathrm{P}+$ & FPR & $\mathrm{Se}$ & $\mathrm{P}+$ & FPR \\
\hline Time features & 94.03 & 94.54 & 99.59 & 5.09 & 99.00 & 25.15 & 2.99 & 85.43 & 66.55 & 2.82 \\
\hline VMD fetaures & 87.03 & 86.64 & 99.43 & 5.77 & 77.36 & 6.41 & 9.95 & 92.87 & 71.91 & 2.86 \\
\hline VMD + Time features & 95.74 & 96.11 & 99.64 & 4.40 & 91.82 & 21.26 & 3.08 & 91.01 & 86.93 & 0.94 \\
\hline
\end{tabular}

Table 4. Results for the inter-patient approach for time features, VMD features and both.

\begin{tabular}{|c|c|c|c|c|c|c|c|c|c|c|}
\hline & \multirow{2}{*}{$\begin{array}{l}\text { Overall } \\
\text { Accuracy }\end{array}$} & \multicolumn{3}{|c|}{$\mathrm{N}$} & \multicolumn{3}{|c|}{$\mathrm{Sv}$} & \multicolumn{3}{|c|}{$\mathrm{V}$} \\
\hline & & $\mathrm{Se}$ & $\mathrm{P}+$ & FPR & $\mathrm{Se}$ & $\mathrm{P}+$ & FPR & $\mathrm{Se}$ & $\mathrm{P}+$ & FPR \\
\hline Time features & 86.93 & 89.47 & 99.05 & 8.57 & 33.77 & 26.67 & 3.04 & 76.35 & 32.75 & 9.94 \\
\hline VMD fetaures & 71.84 & 72.33 & 97.59 & 17.59 & 28.75 & 3.30 & 20.05 & 81.11 & 44.72 & 7.50 \\
\hline VMD + Time features & 92.17 & 92.84 & 99.59 & 3.75 & 72.56 & 25.48 & 6.27 & 91.25 & 80.84 & 1.49 \\
\hline
\end{tabular}

enth Framework Programme (FP7/2007-2013) / ERC Advanced Grant: BIOTENSORS (no 339804)/ TARGID Development of a novel diagnostic medical device to assess gastric motility ]: C32-16-00364/ imec funds 2017. Carolina Varon is a postdoctoral fellow of the Research Foundation-Flanders (FWO). R. Willems is supported as postdoctoral clinical researcher by the Fund for Scientific Research Flanders. This paper reflects only the author's views and the Union is not liable for any use that may be made of the contained information.

\section{References}

[1] de Chazal et al. (2004). Automatic classification of heartbeats using ECG morphology and heartbeat interval features. IEEE Transactions on Biomedical Engineering, 51(7), 1196-1206. https://doi.org/10.1109/TBME.2004.827359

[2] Chun-Cheng Lin et al. (2014), Heartbeat classification using normalized RR intervals and morphological features, Mathematical Problems in Engineering, vol. 2014, Article ID 712474, 11 pages. https://doi.org/10.1155/2014/712474.

[3] Ye, C. et al. (2012). Heartbeat classification using morphological and dynamic features of ECG signals. IEEE Transactions on Biomedical Engineering, 59(10), 2930-2941. https://doi.org/10.1109/TBME.2012.2213253

[4] Lin, C. et al. (2008). Adaptive wavelet network for multiple cardiac arrhythmias recognition. Expert Systems with Applications, 34(4), 2601-2611. https://doi.org/10.1016/J.ESWA.2007.05.008

[5] Rodríguez-Sotelo, J. L. et al. (2010). Weighted-PCA for unsupervised classification of cardiac arrhythmias. 2010 Annual International Conference of the IEEE Engineering in Medicine and Biology Society, EMBC'10, (June 2016), 1906-1909. https://doi.org/10.1109/IEMBS.2010.5627321

[6] Sarfraz, M. et al. (2014). Using Independent Component
Analysis to obtain feature space for reliable ECG arrhythmia classification. In 2014 IEEE International Conference on Bioinformatics and Biomedicine (BIBM) (pp. 62-67). IEEE. https://doi.org/10.1109/BIBM.2014.6999249

[7] Association for the advancement of medical instrumentation. (1998). Testing and reporting performance results of cardiac rhythm and st segment measurement algorithms. ANSI/AAMI EC38, 1998.

[8] Luz, E. J. et al. ECG-based heartbeat classification for arrhythmia detection: A survey. Comput. Methods Programs Biomed. 127, 144-164 (2016).

[9] Vázquez-Seisdedos, C. R. et al. (2013). A comparison of different classifiers architectures for electrocardiogram artefacts recognition. In Iberoamerican Congress on Pattern Recognition (pp. 254-261). Springer, Berlin, Heidelberg.

[10] Dragomiretskiy, K., \& Zosso, D. (2014). Twodimensional Variational Mode Decomposition. IEEE Transactions on Signal Processing, 62(3), 531-544. https://doi.org/10.1109/TSP.2013.2288675.

[11] Guo, Yanfei et al. (2017). An optimized Variational Mode Decomposition for extracting weak feature of viscoelastic sandwich cylindrical structures. Measurement Science and Technology. 29. 10.1088/1361-6501/aa9ef0.

[12] Clifford, G. D. et al. Advanced methods and tools for ECG data analysis. (Artech House, 2006).

[13] Suykens, J. A. K. et al. (2002). Least Squares Support Vector Machines. World Scientific. https://doi.org/10.1142/9789812776655

Address for correspondence:

Amalia Villa

Department of Electrical Engineering (ESAT),

STADIUS, KU Leuven, Belgium

amalia.villagomez@kuleuven.be 